\title{
MICROBIOLOGY AND INFECTIOUS DISEASES
}

November 29-30, 2021 I Webinar

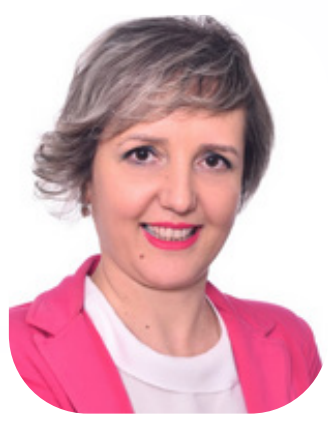

\author{
Oltiana Petri, Albana Daka, Erjona Abazaj, Gentian Huti, Edmond \\ Puca, Blerta Brati
}

American Hospital, Tirana, Albania

\section{The epidemiological situation and clinical characteristic aspect cause by covid-19 in suspected cases in Albania}

Introduction: SARS-CoV-2 has forcefully entered our lives and profoundly changed the perception and habits of the world population, since the December 2019. The aims of this study were to presents the epidemiology data and clinical characteristic aspect of patients suspected with COVID-19 and analyzed in the American laboratory in Tirana, Albania from August 2020 until march 2021.

Methods: This study includes 1205 suspected cases. Professionally trained health workers collected the samples from suspected COVID-19 by using synthetic fiber swabs. Double specimens from nasopharyngeal and pharyngeal swabs were collected from each of person. A standard questionnaire about the demographic and clinical data were filled for each person. The software SPSS version 22.0 was used for analyzed all data and also the examination findings.

Results: The prevalence of SARS Cov-2 resulted to be $31.1 \%$. The average age resulted $42.17 \pm$ 18.6 Stdv with min 1 years old and max 93 age old. The most predominant positive age resulted the age groups $31-40$ years old and $41-50$ years old with positivity $17.3 \%$ and $16.3 \%$ respectively with significant association $p=0.01$. Male were the most analyzed and affected persons $(61.6 \%)$ in this study compared with female (38.4\%), with a strong significant association $p$ value $=0.0004$. It turned out that more than half of patients refereed at least two symptoms in the same time of analyze and about $1 / 3$ refereed more than three symptoms. Our searches found an association for presence of all typical symptoms with positivity except the temperature. We identified that for all of these symptoms the $p$ value resulted less than 0.05 .

Conclusion: ARS Cov-2 in the early stages of the disease, is highly contagious for this reason the screening of people with symptoms is recommended to determine who should be quarantined and be tested. In this manner we can decrease the rate of infection and protect healthy people from infection.

Key words: SARS-CoV-2, epidemiological situation, American Hospital patients 